\title{
Procedimiento de gestión para asegurar la calidad de una universidad. Caso de estudio Universidad Técnica de Manabí
}

\author{
Management procedure to ensure the quality of a university. \\ Case study Technical University of Manabí
}

\author{
Vicente Félix Veliz Briones ${ }^{1 *} \quad$ Alicia Alonso Becerra $^{2} \quad$ Daniel Alfonso Robaina $^{3}$ \\ María Sonia Fleitas Triana ${ }^{2} \quad$ Ester Michelena Fernández ${ }^{2}$ \\ Recibido 7 de mayo de 2018, aceptado 29 de Octubre de 2018 \\ Received: May 7, 2018 Accepted: October 29, 2018
}

\begin{abstract}
RESUMEN
La necesidad de incrementar los niveles de calidad de las universidades principalmente en el ámbito latinoamericano ha permitido que se desarrollen modelos para la evaluación de las instituciones de educación superior y para la acreditación de los programas que en ella se desarrollan. Sin embargo, estos modelos no deben actuar en las universidades puntualmente sino que deben formar de la planificación estratégica y del sistema de gestión de la universidad para que de forma sistemática se contribuya al cumplimiento de los criterios e indicadores que forman parte del patrón de calidad. El procedimiento propuesto tiene el objetivo de encontrar la adecuada correspondencia entre el modelo de evaluación y acreditación y el sistema de gestión de la universidad. La aplicación del procedimiento en la Universidad Técnica de Manabí contribuyó a que esta universidad mejorara los niveles de acreditación nacional desde la categoría "D" hasta la "B" y la visibilidad internacional.
\end{abstract}

Palabras clave: Gestión de la calidad universitaria, gestión por procesos, dirección estratégica.

\begin{abstract}
The need to increase the quality levels of universities, mainly in Latin America, has allowed the development of models for the evaluation of higher education institutions and for the accreditation of the programs that are developed in it, however, Models should not act in the universities on time but should form the strategic planning and management system of the university so that systematically contribute to compliance with the criteria and indicators that are part of the quality pattern. The proposed procedure has the objective of finding the appropriate correspondence between the evaluation and accreditation model and the management system of the university. The application of the procedure in the Technical University of Manabi contributed to that this university improved the levels of national accreditation from category " $D$ " to " $B$ " and the international visibility.
\end{abstract}

Keywords: University quality management, process management, strategic management.

1 Universidad Técnica de Manabí. Portoviejo, Manabí. Ecuador. E-mail: vicenteveliz@ gmail.com

2 Universidad Tecnológica de La Habana “José Antonio Echeverría”, CUJAE. Departamento de Ingeniería Industrial. Marianao, La Habana.Cuba.E-mail: alonso@tesla.cujae.edu.cu; sfleitas@ind.cujae.edu.cu; emichele@tesla.cujae.edu.cu

3 Universidad Tecnológica de La Habana "José Antonio Echeverría", CUJAE. Centro de Estudios de Técnicas de Dirección. Marianao, La Habana. Cuba. dalfonso@ind.cujae.edu.cu

* Autor de Correspondencia: vicenteveliz@gmail.com 


\section{INTRODUCCIÓN}

En la primera y segunda décadas de este siglo se reconocen cambios en la Educación Superior en el contexto latinoamericano asociados en lo fundamental a: la creciente heterogeneidad y diversidad de instituciones, el surgimiento de las macro universidades y de las instituciones de formación técnica, el aumento del número de estudiantes y del sistema privado, el incremento de la investigación científica, el impacto de las nuevas tecnologías, la mercantilización de la educación, el desarrollo de nuevas carreras y de nuevas áreas de conocimiento de base interdisciplinaria. Estos cambios han hecho necesario establecer en cada país mecanismos de control que contribuyan a garantizar el cumplimiento de un conjunto de indicadores "representativos" de la calidad de las instituciones, que se han expresado en sistemas nacionales y externos de evaluación y acreditación de la calidad.

De acuerdo a $[13,8]$ el aumento de la calidad de la educación superior deja de ser un proceso unilineal que puede ser evaluado por un conjunto de indicadores estáticos y cuantitativos. Por el contrario, aparece como un proceso multifactorial cuyos resultados difieren dependiendo de los patrones de referencia. Todo ello se manifiesta en que los resultados de un cambio en la calidad de la educación pueden ser considerados muy positivos para aquéllos que comparten ciertos valores y muy negativos para quienes sustentan valores antagónicos.

Por otro lado, uno de los elementos utilizados en la actualidad para comparar y delimitar la excelencia son los rankings universitarios [4, 14-15, 17]. Se ha observado que los rankings buscan medir una institución a través de un solo indicador compuesto, generando una lista ordenada de instituciones con un determinado valor, en donde se establece una posición o una medida ponderada $[9,5]$.

Sin embargo, los países tienen características y niveles de desarrollo diferentes y las misiones de las universidades deben responder a las necesidades de la sociedad en que desarrollan su labor, que pueden ser muy diversas. Por lo anteriormente expresado, deben generar resultados de forma intencionada que, cumpliendo con su misión, permitan medir su desempeño acorde a los patrones de calidad nacionales e internacionales [6, 11]. En la literatura consultada sobre gestión universitaria [1-3], se reconoce la gestión por procesos, como el enfoque a utilizar en los sistemas de gestión universitaria.

El enfoque a procesos es una herramienta de gestión que puede ser utilizada para resolver la discrepancia existente entre la diversidad de las universidades y los sistemas de medición para evaluar y acreditar la calidad.

\section{MÉTODO}

El método utilizado para la realización del procedimiento de gestión para asegurar la calidad de una Universidad consiste en la integración de la gestión por procesos con los criterios de los patrones de calidad de la Educación Superior y los rankings internacionales. En la Figura 1 aparecen las etapas a seguir.

\section{Etapa I. Identificación y selección de los indicadores de los patrones de calidad}

El objetivo de esta etapa es poder hacer un estudio de diferentes patrones de calidad de la Educación

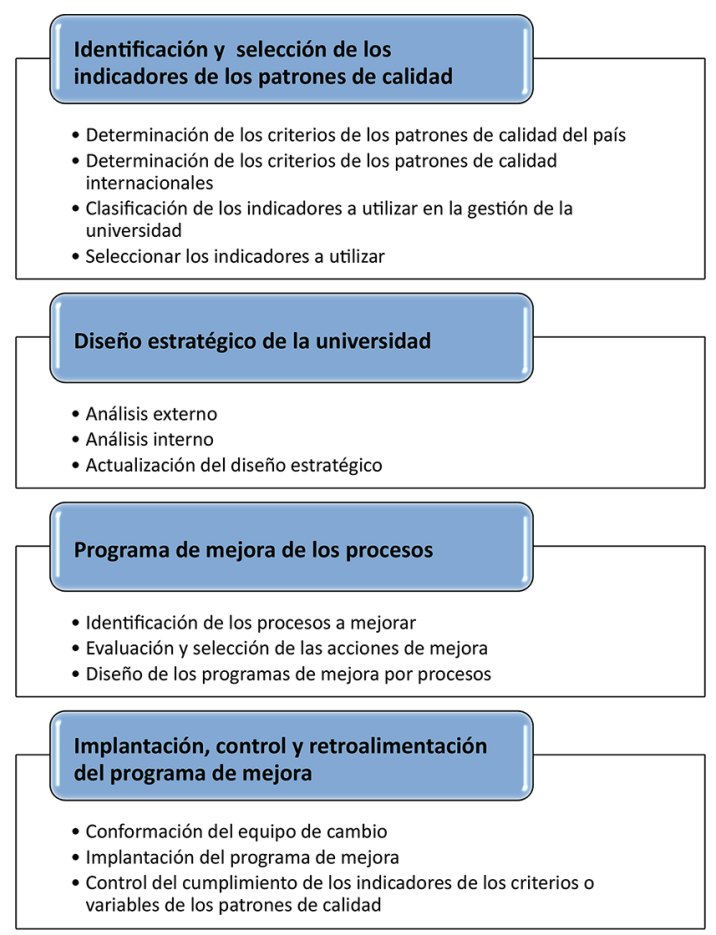

Figura 1. Etapas del procedimiento de gestión para asegurar la calidad de una universidad. 
Superior, de manera que se realice un levantamiento de información y una actualización del entorno donde opera la Universidad. Teniendo en cuenta los puntos de vistas de la alta dirección y la capacidad de operaciones de la Universidad se seleccionan los criterios, subcriterios e indicadores de los patrones de calidad de la Educación Superior que la Universidad se propone gestionar desde sus procesos para asegurarlos y alcanzar resultados positivos en ese contexto.

\section{Paso 1.1. Determinación de los criterios del patrón de calidad del país}

Determinar los criterios, subcriterios e indicadores del modelo de evaluación de las universidades en el país y establecimiento de las metas requeridas para alcanzar niveles de evaluación superior en dicho modelo, para lo cual es necesario conocer los niveles y valor del cumplimiento de las universidades que ostentan la categoría que se desea alcanzar, se realiza un estudio de referencia.

Paso 1.2. Determinación de los criterios de los patrones de calidad y rankings internacionales Determinar los modelos internacionales de evaluación institucional de las universidades y comparar sus criterios, subcriterios e indicadores con los que contiene el modelo del país, con el objetivo de identificar los que coinciden y los que solo aparecen en los modelos externos. Además, se analiza el comportamiento de los indicadores de los rankings internacionales, así como los sitios de gestión académica y de la ciencia. Con ello se pueden proponer los indicadores a seleccionar para garantizar la mejora de los procesos para asegurar la calidad en la Universidad.

\section{Paso 1.3. Clasificación de los indicadores a utilizar en la gestión de la universidad}

En este paso se clasifican los indicadores en dos grupos.

- Grupo 1: Los que aparecen en el patrón de evaluación institucional del país y al menos uno de los internacionales analizados.

- Grupo 2: Los que solo aparecen en el modelo del país.

Con la clasificación por grupo de los indicadores se establece un criterio para poder seleccionar los indicadores a gestionar para asegurar la calidad y mejorar la categoría de acreditación, aunque es necesario tener en cuenta el criterio de la dirección de la universidad para hacer la selección definitiva.

Paso 1.4. Seleccionar los indicadores a utilizar Teniendo en cuenta la clasificación anterior se seleccionarán los indicadores a tener en cuenta para la actualización de los procesos como parte del diseño estratégico.

\section{Etapa II. Diseño estratégico de la Universidad}

El objetivo de esta etapa está orientado a actualizar el diseño estratégico de la Universidad teniendo en cuenta los criterios, subcriterios e indicadores de los patrones de calidad seleccionados. Los pasos que se proponen responden a un diagnóstico estratégico contextualizado al objetivo del Modelo de gestión universitaria por procesos para asegurar la calidad de la universidad.

\section{Paso 2.1. Análisis externo}

En el análisis externo se propone realizar un estudio de los diferentes actores del entorno de la Universidad teniendo en cuenta las variables siguientes: económica, socio cultural, político legal, tecnológica y ecológica. Valorar las influencias de estas variables en la gestión de la Universidad para alcanzar los criterios de los patrones de calidad de la educación superior, si su influencia es positiva se clasifica como oportunidad y si es negativa como amenaza. En la Tabla 1 se presenta la matriz para el tratamiento de esta información.

En la matriz se presenta por las filas las clasificaciones del tipo de variable según lo económico, social cultural, político legal, tecnológico y ecológico. En las columnas aparecen las variables identificadas en el entorno de la Universidad. En cada celda, en donde coincida la variable y la clasificación, se presenta la evaluación del nivel de impacto de la variable a la Universidad en función de asegurar los criterios de los Patrones de Calidad de la Educación Superior seleccionados.

- Si la variable impacta de manera positiva, se pone el signo $(+)$.

- Si la variable impacta de manera negativa, se pone el signo (-).

El signo es para identificar el tipo de impacto, cuando es positivo se considera una oportunidad y cuando 
Tabla 1. Análisis de la influencia de las variables del entorno en la gestión de la Universidad.

\begin{tabular}{|c|c|c|c|c|c|}
\hline \multirow{2}{*}{ Variables del Entorno } & \multicolumn{5}{|c|}{ Clasificación } \\
\cline { 2 - 6 } & Económica & Social cultural & Político legal & Tecnológica & Ecológica \\
\hline $\mathrm{V}_{1}$ & NI & NI & NI & NI & NI \\
\hline $\mathrm{V}_{2}$ & NI & NI & NI & NI & NI \\
\hline Vn & NI & NI & NI & NI & NI \\
\hline
\end{tabular}

Leyenda:

V: variables identificadas en el entorno.

NI: nivel de impacto de la variable del entorno.

es negativo se considera una amenaza. El nivel de influencia está dado por la clasificación siguiente:

- 5: impacto alto.

- 3: impacto medio.

- 1 : impacto bajo.

La escala seleccionada tiene tres niveles para lograr una clasificación inequívoca del impacto y los pesos asignados a cada nivel se establecen a partir de valores que sea sencillo trabajar con ellos. Contar con un rango de respuestas también permitirá identificar fácilmente las áreas de mejora. La escala tiene un número de niveles impares para poder obtener un punto medio. Para la evaluación del nivel de impacto se propone realizar ejercicios grupales con la alta dirección de la universidad.

\section{Paso 2.2. Análisis interno}

En este paso se tiene en cuenta el análisis de las capacidades de la Universidad para gestionar sus procesos y las exigencias de los criterios de los Patrones de Calidad de la Educación Superior. En la Tabla 2 se propone una matriz para apoyar este análisis.

En la matriz, en las filas se presentan los procesos de la Universidad y se relacionan con las columnas que son los criterios de los Patrones de Calidad de la Educación Superior seleccionados. En cada celda se evalúa el desempeño de la capacidad del proceso para asegurar los criterios de los Patrones de Calidad.

- Si existe desempeño y este es alto o bueno se considera fortaleza, si existe desempeño, pero no es alto o bueno no se considera fortaleza.

Para la valoración del desempeño se tiene la escala siguiente:

- 5: Desempeño Alto de la capacidad del proceso para asegurar el criterio del patrón de calidad.

- 3: Desempeño Medio de la capacidad del proceso para asegurar el criterio del patrón de calidad.

- 1: Desempeño Bajo de la capacidad del proceso para asegurar el criterio del patrón de calidad.

- No hay relación del proceso con el criterio del patrón de calidad.

\section{Paso 2.3. Actualización el diseño estratégico}

La Universidad debe orientar el diseño estratégico teniendo en cuenta el nivel de acreditación que se proponen alcanzar acorde a los patrones de calidad que decide gestionar y los resultados obtenidos

Tabla 2. Análisis del desempeño de las capacidades de la Universidad.

\begin{tabular}{|c|c|c|c|c|c|c|}
\hline \multirow{4}{*}{ Procesos } & \multicolumn{4}{|c|}{ Criterios de los Patrones de Calidad de la Educación Superior } \\
\cline { 2 - 7 } & \multicolumn{2}{|c|}{ CPCES 1 } & \multicolumn{2}{c|}{ CPCES 2 } & \multicolumn{2}{c|}{ CPCES n } \\
\cline { 2 - 7 } & Capacidad & Desempeño & Capacidad & Desempeño & Capacidad & Desempeño \\
\hline P1 & & & & & & \\
\hline P2 & & & & & & \\
\hline Pn & & & & & & \\
\hline
\end{tabular}


en el diagnóstico estratégico externo e interno, en el contexto que está actuando, lo que garantizará que en el diseño estratégico se tenga en cuenta las particularidades de cada Universidad y los aspectos exigidos en dicho patrones. Los componentes a tener en cuenta en el diseño estratégico son: misión, visión, valores, objetivos estratégicos, procesos y estrategias

A través de la gestión de los procesos, la Universidad debe alcanzar un mayor cumplimiento de los Patrones de Calidad de la Educación Superior y de esta manera un reconocimiento superior tanto a nivel nacional como internacional. En la Figura 2 se muestra como a través de la gestión por procesos, la Universidad puede ir mejorando su desempeño y con ello obtener niveles de acreditación superiores. En el caso de Ecuador transitar de la categoría D ( sin reconocimiento nacional) a la categoría A (excelencia nacional) pudiendo llegar al reconocimiento en los rankings internacionales.

A partir de la misión y visión determinadas se podrá identificar y determinar los procesos de la universidad, que condicionan el alcance del sistema de gestión, clasificándolos y definiendo las interrelaciones internas y externas para construir el mapa de procesos.

Las estrategias a utilizar son clasificadas de acuerdo a los diferentes niveles de reconocimientos que aspira obtener la Universidad de la forma siguiente:

1. Estrategia de sobrevivir

2. Estrategia de fortalecer

3. Estrategia avanzar y desarrollar
El criterio de selección de las acciones a aplicar por cada estrategia se basa en dos variables: el Impacto (I) y la Viabilidad (V), esta última como función del costo (C) y del tiempo (T).

Impacto (I): Nivel con que inciden las acciones en las mejoras de los procesos para asegurar los patrones de calidad.

Viabilidad (V): Valoración sobre la posibilidad de realizar las acciones de las mejoras de los procesos para asegurar los patrones de calidad, teniendo en cuenta el costo de la acción y el tiempo de ejecución.

\section{Estrategia de Sobrevivir:}

Este tipo de estrategia está orientada a garantizar los indicadores mínimos requeridos para la existencia y el funcionamiento de la Universidad a partir de desarrollar alternativas de trabajo que permitan de manera rápida hacer mejoras en la Universidad.

En la estrategia de sobrevivir la viabilidad tiene mayor peso, ya que se necesitan mejoras cuyos resultados puedan lograrse en un período menor de tiempo, lo que demore en alcanzarse, no es factible, ya que el resultado se necesita en el corto plazo, de lo contrario la Universidad corre el riesgo de no poder continuar funcionando.

En esta etapa la mayor parte de las mejoras deben estar asociadas a los proyectos de organización y de infraestructura y la decisión se toma evaluando la expresión siguiente:

$$
\text { Estrategia de Sobrevivir= 0,4 I + 0,6 V }
$$

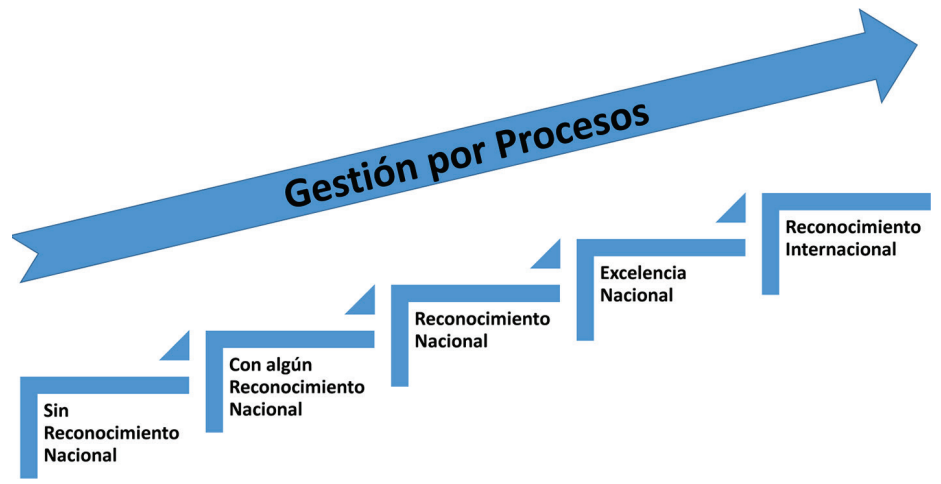

Figura 2. Gestión universitaria por procesos para asegurar la mejora de los niveles de acreditación. 


\section{Estrategia de Fortalecer:}

Está orientada al logro de un funcionamiento estable de la Universidad prestando atención al aspecto académico, especialmente en la actividad de pregrado.

En la estrategia de fortalecer la variable impacto tiene mayor peso, ya que se necesitan mejoras cuyos resultados tengan un impacto significativo a mediano y largo plazo, aunque la solución demore más.

En esta etapa las mejoras están asociadas al desarrollo de los recursos humanos de la Universidad. La decisión se toma evaluando la expresión siguiente:

$$
\text { Fortalecer }=0,6 \mathrm{I}+0,4 \mathrm{~V}
$$

\section{Estrategia de Avanzar y Desarrollar:}

Está orientada a niveles superiores en el trabajo docente e investigativo en la universidad, prestando especial atención al desarrollo de la actividad de postgrado y la producción científica de la universidad.

En la estrategia de avanzar y desarrollar aumenta el peso de la variable impacto, ya que la universidad necesita mejoras cuyos resultados permitan alcanzar la excelencia de la institución y el reconocimiento internacional. Las soluciones en esta etapa son más complejas y se alcanzan en un mayor plazo de tiempo, consolidando el enfoque de gestión propuesto en el modelo de gestión universitaria por procesos para asegurar la calidad de la universidad.

En esta etapa la mayor parte de las mejoras están asociadas a los proyectos de gestión de conocimiento y producción de capital intelectual de la Universidad y la consolidación de la gestión por procesos. $\mathrm{La}$ decisión se toma evaluando la expresión siguiente.

$$
\text { Avanzar y desarrollar }=0,8 \mathrm{I}+0,2 \mathrm{~V}
$$

En el establecimiento de las estrategias se tiene en cuenta como criterios principales la capacidad de la
Universidad en el momento analizado y los criterios del patrón de calidad de la Educación Superior que se quiere garantizar. En la Tabla 3 se propone como evaluar la capacidad de la Universidad en las etapas siguientes: Sin reconocimiento nacional, reconocimiento nacional y excelencia nacional con reconocimiento internacional.

Una Universidad con capacidad baja comenzando a desplegar estrategias de Sobrevivir, debe crear nuevas capacidades en la organización para poder implementar estrategias de Fortalecer y posteriormente estrategias de Avanzar y Desarrollar.

\section{Etapa III. Programa de mejoras de los procesos} El objetivo de esta etapa es establecer las mejoras de las actividades de los procesos de la Universidad teniendo en cuenta las estrategias que se han establecido.

\section{Paso 3.1. Identificación de los procesos de la Universidad a mejorar}

Para cada uno de los procesos determinados hay que analizar el desempeño actual de la universidad y la meta a alcanzar para cumplir con el patrón de calidad como se muestra en la Tabla 4, este análisis se hará por indicadores.

Con los resultados de este análisis se determina los procesos que tienen mayor impacto en el cumplimiento de los indicadores y que deben ser mejorados. Los campos que deben estar determinados en el Plan de Mejora son: proceso, acciones, tareas, fecha de inicio, fecha de terminación, responsable, presupuesto e indicadores.

Paso 3.2 Evaluación y selección de las acciones de mejoras

Para la evaluación de las mejoras se tiene en cuenta los criterios planteados en la Etapa II, Paso 2.3 de Impacto (I) y Viabilidad (V) como función del costo

Tabla 3. Matriz de tipos de estrategias en la Universidad.

\begin{tabular}{|c|c|c|c|c|}
\hline \multirow{2}{*}{$\begin{array}{c}\text { Capacidad de } \\
\text { la Universidad }\end{array}$} & $\begin{array}{c}\text { Sin reconocimiento } \\
\text { nacional }\end{array}$ & $\begin{array}{c}\text { Reconocimiento } \\
\text { nacional }\end{array}$ & $\begin{array}{c}\text { Excelencia } \\
\text { nacional }\end{array}$ & $\begin{array}{c}\text { Reconocimiento } \\
\text { internacional }\end{array}$ \\
\hline Baja & Sobrevivir & & & \\
\hline Media & & Fortalecer & & \\
\hline Alta & & & Avanzar y desarrollar & Avanzar y desarrollar \\
\hline
\end{tabular}


Tabla 4. Matriz de relación entre los entre los criterios, subcriterios e indicadores con los procesos.

\begin{tabular}{|c|c|c|c|c|c|}
\hline Criterio & Sub-criterio & Indicadores & Fórmula de cálculo & Meta & Proceso \\
\hline \multirow{9}{*}{ Criterio 1} & \multirow{3}{*}{ Subcriterio 1} & Indicador 1 & & & \\
\hline & & Indicador 2 & & & \\
\hline & & Indicador $\mathrm{n}$ & & & \\
\hline & \multirow{3}{*}{ Subcriterio 2} & Indicador 1 & & & \\
\hline & & Indicador 2 & & & \\
\hline & & Indicador $\mathrm{n}$ & & & \\
\hline & \multirow{3}{*}{ Subcriterio $\mathrm{n}$} & Indicador 1 & & & \\
\hline & & Indicador 2 & & & \\
\hline & & Indicador $\mathrm{n}$ & & & \\
\hline \multirow{9}{*}{ Criterio 2} & \multirow{3}{*}{ Subcriterio 1} & Indicador 1 & & & \\
\hline & & Indicador 2 & & & \\
\hline & & Indicador $\mathrm{n}$ & & & \\
\hline & \multirow{3}{*}{ Subcriterio 2} & Indicador 1 & & & \\
\hline & & Indicador 2 & & & \\
\hline & & Indicador $\mathrm{n}$ & & & \\
\hline & \multirow{3}{*}{ Subcriterio $n$} & Indicador 1 & & & \\
\hline & & Indicador 2 & & & \\
\hline & & Indicador $\mathrm{n}$ & & & \\
\hline \multirow{9}{*}{ Criterio $\mathrm{n}$} & \multirow{3}{*}{ Subcriterio 1} & Indicador 1 & & & \\
\hline & & Indicador 2 & & & \\
\hline & & Indicador $\mathrm{n}$ & & & \\
\hline & \multirow{3}{*}{ Subcriterio 2} & Indicador 1 & & & \\
\hline & & Indicador 2 & & & \\
\hline & & Indicador $\mathrm{n}$ & & & \\
\hline & \multirow{3}{*}{ Subcriterio $n$} & Indicador 1 & & & \\
\hline & & Indicador 2 & & & \\
\hline & & Indicador $\mathrm{n}$ & & & \\
\hline
\end{tabular}

(C) y del tiempo (T). Para evaluar la Importancia y Viabilidad se toman los criterios de la alta dirección de la Universidad, se trabajará en una escala de uno (1) a diez (10) donde 1 es el menor valor y 10 el mayor valor. Ejemplo: 1 es la de menor importancia y 10 la más importante; 1 es la menos viable y 10 la más viable.

La selección se realizará por las concepciones planteadas en cada estrategia en la etapa anterior.
En la Tabla 5 se propone un panel para realizar este análisis.

Paso 3.3. Diseño de los programas de mejora por procesos

Para diseñar las mejoras se tienen en cuenta las capacidades de la Universidad y los criterios del patrón de calidad que se quiere alcanzar. En la Tabla 6 se identifican los proyectos de mejora que se desarrollarán y el proceso o los procesos que se mejorarán, el alcance

Tabla 5. Panel de análisis de mejoras de los procesos.

\begin{tabular}{|l|c|c|c|l|l|l|}
\hline Proceso & $\begin{array}{c}\text { Acción de } \\
\text { mejora }\end{array}$ & $\begin{array}{c}\text { Impacto } \\
(\mathbf{1 - 1 0})\end{array}$ & $\begin{array}{c}\text { Viabilidad } \\
(\mathbf{1 - 1 0})\end{array}$ & Estrategia & $\begin{array}{c}\text { Evaluación } \\
\text { de la acción }\end{array}$ & $\begin{array}{c}\text { Selección } \\
\text { (si o no) }\end{array}$ \\
\hline & & & & & & \\
\hline & & & & & & \\
\hline
\end{tabular}


Tabla 6. Presentación de las mejoras de las actividades de los procesos de la Universidad.

\begin{tabular}{|l|l|l|l|l|l|l|l|}
\hline Proceso & Acciones & Tareas & Fecha Inicio & Fecha Terminación & Responsable & Presupuesto & Indicadores \\
\hline & & & & & & & \\
\hline & & & & & & & \\
\hline & & & & & & & \\
\hline
\end{tabular}

de la mejora, el responsable, los participantes, el tiempo de ejecución y los recursos necesarios.

Los programas de mejora a desarrollar en la Universidad en el camino a la excelencia y el reconocimiento internacional dependen de la etapa en que se encuentra en el momento de comenzar a aplicar el procedimiento. El período para el tránsito entre una etapa y la superior se incrementa en la medida en que mayor sea la categoría debido a que los resultados que se requieren para alcanzar los niveles superiores están asociados a programas de mejora de gestión del conocimiento que demoran más tiempo para su ejecución y logro de resultados. Al llegar a la tercera etapa de tránsito hacia la excelencia y el reconocimiento internacional de la Universidad, cuando se desarrollan los programas de mejora de Gestión de conocimiento y producción de capital intelectual se hace necesario consolidar la gestión por procesos. Todos los programas de mejora que se desarrollen deben ir acompañados de programas de capacitación.

\section{Etapa IV. Implantación, control y retroalimentación del programa de mejoras}

En esta etapa es donde se implementan los cambios o las mejoras en la Universidad por lo que es importante tener un equipo que lidere esas trasformaciones y un sistema que permita comprobar o verificar el aseguramiento de los patrones de calidad a partir de los procesos.

\section{Paso 4.1. Conformación del equipo de cambio}

Para conducir el proceso de cambio se conforma un equipo formado por directivos y docentes con diversos conocimientos y experiencia práctica en actividades de gestión universitaria. Este equipo debe ser preparado sobre el modelo, las herramientas y el enfoque de gestión universitaria teniendo en cuenta el cumplimiento de los criterios de los patrones de calidad a través de los procesos. Este equipo es quien debe guiar la implantación de los programas de mejoras.
Paso 4.2. Implantación del programa de mejoras En este paso hay que ejecutar el programa de mejoras que fue diseñado en el Paso 3.3, para ello hay que dirigir el esfuerzo común de los subordinados para alcanzar las metas y objetivos de la organización mediante la orientación, la comunicación, la motivación, la preparación y la supervisión de los involucrados.

Paso 4. 3. Control del cumplimiento de los indicadores de los criterios o variables de los patrones de calidad En la Figura 3 se muestra los niveles de control y evaluación que deben cumplimentarse. Los indicadores a nivel de los procesos, que ya están asociados a los indicadores de los patrones de calidad, y a nivel de la organización se evalúan anualmente para analizar el cumplimiento de los objetivos estratégicos.

El análisis se puede realizar utilizando la Tabla 7 en la cual se considera el cumplimiento de los indicadores y la evolución que tienen en el tiempo para alcanzar las metas.

La información de la Tabla 7 anterior se puede filtrar por:

- Procesos: para evaluar el cumplimiento de los indicadores por cada proceso.

- Criterios y subcriterios: para evaluar el cumplimiento de los indicadores del patrón de calidad.

Sistema de Control de Gestión

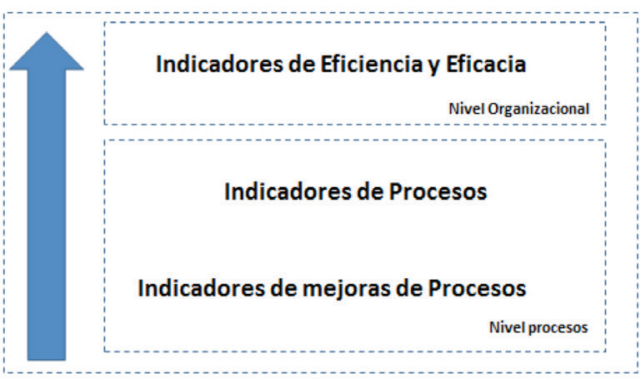

Figura 3. Sistema de control de gestión. 
Tabla 7. Cumplimiento de los indicadores y su evolución en el tiempo.

\begin{tabular}{|c|c|c|c|c|c|c|c|}
\hline \multicolumn{3}{|c|}{ Patrón de Calidad } & Meta & \multicolumn{3}{|c|}{ Cumplimiento } & \multirow[t]{2}{*}{ Procesos } \\
\hline Criterios & Sub-criterios & Indicadores & & Año 1 & Año 2 & Añon & \\
\hline \multirow{9}{*}{ Criterio 1} & \multirow{3}{*}{ Subcriterio 1} & Indicador 1 & & & & & \\
\hline & & Indicador 2 & & & & & \\
\hline & & Indicador $\mathrm{n}$ & & & & & \\
\hline & \multirow{3}{*}{ Subcriterio 2} & Indicador 1 & & & & & \\
\hline & & Indicador 2 & & & & & \\
\hline & & Indicador $\mathrm{n}$ & & & & & \\
\hline & \multirow{3}{*}{ Subcriterio $n$} & Indicador 1 & & & & & \\
\hline & & Indicador 2 & & & & & \\
\hline & & Indicador $\mathrm{n}$ & & & & & \\
\hline \multirow{9}{*}{ Criterio 2} & \multirow{3}{*}{ Subcriterio 1} & Indicador 1 & & & & & \\
\hline & & Indicador 2 & & & & & \\
\hline & & Indicador $\mathrm{n}$ & & & & & \\
\hline & \multirow{3}{*}{ Subcriterio 2} & Indicador 1 & & & & & \\
\hline & & Indicador 2 & & & & & \\
\hline & & Indicador $\mathrm{n}$ & & & & & \\
\hline & \multirow{3}{*}{ Subcriterio $n$} & Indicador 1 & & & & & \\
\hline & & Indicador 2 & & & & & \\
\hline & & Indicador $\mathrm{n}$ & & & & & \\
\hline \multirow{9}{*}{ Criterio $\mathrm{n}$} & \multirow{3}{*}{ Subcriterio 1} & Indicador 1 & & & & & \\
\hline & & Indicador 2 & & & & & \\
\hline & & Indicador $\mathrm{n}$ & & & & & \\
\hline & \multirow{3}{*}{ Subcriterio 2} & Indicador 1 & & & & & \\
\hline & & Indicador 2 & & & & & \\
\hline & & Indicador $\mathrm{n}$ & & & & & \\
\hline & \multirow{3}{*}{ Subcriterio $\mathrm{n}$} & Indicador 1 & & & & & \\
\hline & & Indicador 2 & & & & & \\
\hline & & Indicador $\mathrm{n}$ & & & & & \\
\hline
\end{tabular}

\section{DISCUSIÓN}

En el análisis bibliográfico realizado, se evidenció la contradicción existente entre los criterios o variables de los modelos de acreditación universitaria y la gestión por procesos, así como la necesidad de establecer un Procedimiento de gestión para asegurar la calidad de la Universidad.

Para garantizar el mejoramiento continuo de la calidad de una Universidad, obtener categorías superiores de acreditación nacional y reconocimiento internacional, se diseñó el Procedimiento de gestión para asegurar la Calidad de una Universidad.

El Procedimiento propuesto, desde el diseño de los procesos, considera los criterios o variables de los patrones de calidad de la educación superior y de los rankings internacionales.
De acuerdo a los diferentes niveles de reconocimientos que aspira a obtener la Universidad en cada momento se definieron los tres tipos de estrategias: sobrevivir, fortalecer y avanzar - desarrollar.

El procedimiento de gestión para asegurar la calidad de una Universidad fue implantado en la Universidad Técnica de Manabí. Fueron diseñadas las acciones de mejoras para cada una de los tres tipos de estrategias, seleccionándose para su implantación las dieciocho que más contribuyen a asegurar la calidad de la Universidad (teniendo en cuenta los criterios de Impacto y Viabilidad), las que están asociadas a cinco procesos (Académico, Investigaciones - posgrado, Vinculación con la sociedad, Informatización e Infraestructura). [16].

El $100 \%$ de los indicadores que tienen impacto directo en las mejoras de los procesos de la UTM 
presentan resultados superiores en el 2015 con respecto al 2013.

En cuanto a los indicadores cuantitativos se presentan de manera gráfica los comportamientos de los mismos por subcriterios. En la Figura 4, Figura 5 y Figura 6 se observa el avance en el 2015 con respecto al 2013 en los indicadores: Formación de Posgrado, Doctores a Tiempo Completo y Posgrado en formación del Subcriterio de Posgrado. [6-7].

En cuanto al subcriterio Dedicación en la Figura 7 se observan resultados superiores en el 2015 con respecto al 2013 en los indicadores de Estudiantes por Profesor Tiempo Completo, Horas Clase Promedio por Profesor Tiempo Completo y Horas Clase Promedio por Profesor Medio Tiempo (MT) / Tiempo Parcial (TP).

En la Figura 8 se observan valores superiores del 2015 con respecto al 2013 en indicadores del subcriterio Resultados de Investigación como son: Producción Científica y Producción Regional.

En el caso de los subcriterios de Infraestructura y TIC en la Figura 9 se observan avances en el 2015 con respecto al 2013 en los indicadores de Infraestructura: Calidad de Aula y en el caso de las TIC el indicador Conectividad.

En el diseño de la investigación se planteó que el cumplimiento de la hipótesis se realizará a partir de la evaluación de las variables siguientes:

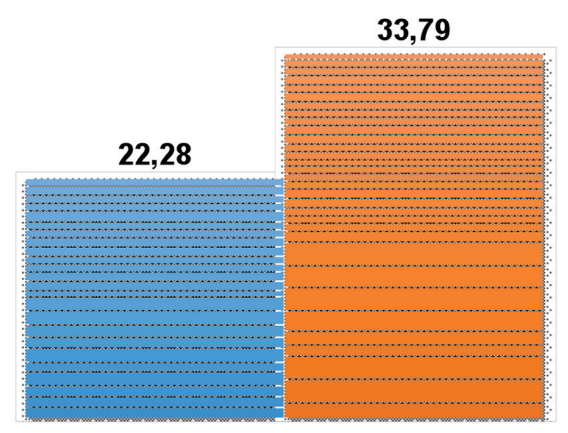

FORMACION POSGRADO

\section{Postgrado \\ 20132015}

Figura 4. Evaluación del indicador Formación de Posgrado.

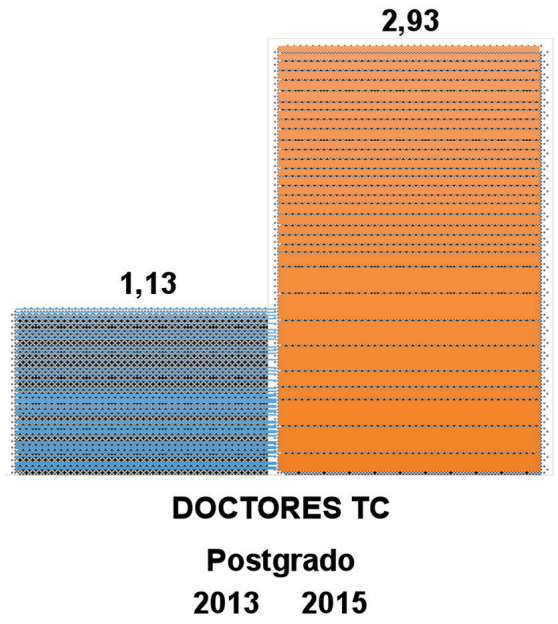

Figura 5. Evaluación del indicador Doctores TC.

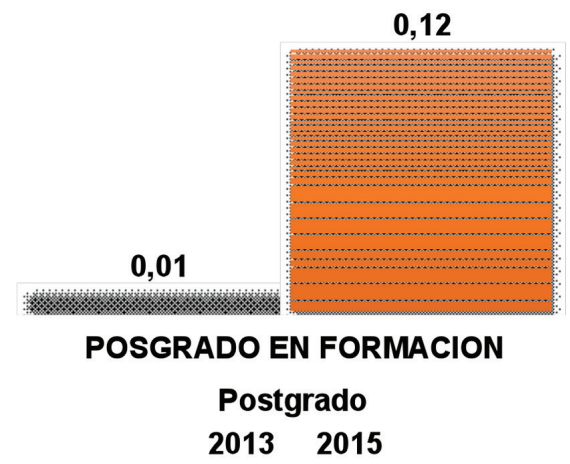

Figura 6. Evaluación del indicador Posgrado en formación.

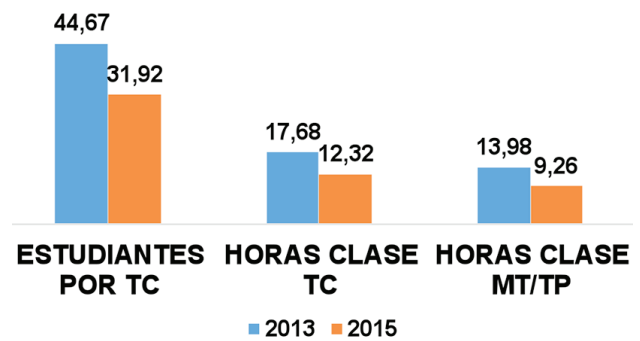

Figura 7. Evaluación del indicador Dedicación.

- Variable independiente: Sistema de gestión

- Variable dependiente: Evaluación externa

En la Tabla 8 se muestra el comportamiento de las variables dependiente e independiente. 


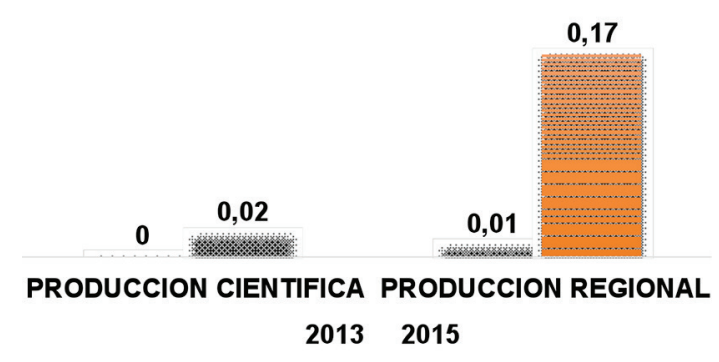

Figura 8. Evaluación de indicadores del subcriterio: Resultados de Investigación.

0,12

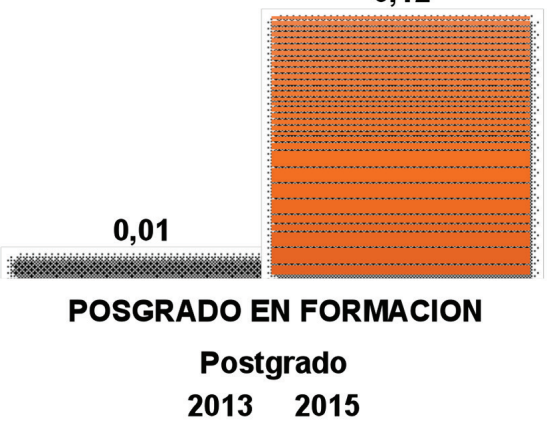

Figura 9. Evaluación de los indicadores del subcriterio: Infraestructura y TIC.

\section{CONCLUSIONES}

El modelo de gestión universitaria por procesos para asegurar la calidad de la Universidad y el procedimiento general para su aplicación garantizan el mejoramiento continuo de la calidad del desempeño de la Universidad y obtener categorías superiores de acreditación nacional y reconocimiento internacional.

El modelo de gestión universitaria propuesto logra una adecuada correspondencia entre la planificación estratégica, el sistema de gestión, los criterios o variables de los patrones de calidad de la educación superior y de los rankings internacionales.

El $100 \%$ de los indicadores de los criterios del modelo de evaluación y acreditación de Ecuador, que tienen impacto directo en las mejoras de los procesos, presentaron resultados superiores en el 2015 con respecto al 2013 en la Universidad Técnica de Manabí.

La implantación del modelo de gestión por procesos para asegurar la calidad en la Universidad Técnica de Manabí contribuyó a que la Universidad mejorara los niveles de acreditación nacional desde la categoría "D" a la "B" y la visibilidad internacional.

\section{REFERENCIAS}

[1] A. Alonso, E. Michelena y D. Alfonso. "Dirección de una Universidad 16 Convención Científica de Ingeniería y Arquitectura". La Habana, Cuba, 2012. ISBN 978-959-261-405-5.

[2] A. Alonso, E. Michelena \& D. Alfonso. "Dirección por procesos en la Universidad". Revista de Ingeniería Industrial, XXXIV(1): 87-95. Enero, 2013. ISSN 1815-5936.

[3] A. Alonso, O. Llanes, E. Michelena, M.S. Fleitas y R. Serra. "Gestión de la ciencia en la universidad: caso de estudio CUJAE". Ingeniare. Revista Chilena de Ingeniería. Vol. $25 \mathrm{~N}^{\circ}$ 2, pp. 277-288. ISSN: 0718-3305 (versión en línea). 2017.

[4] O. Barsky. "Acerca de los rankings internacionales de las universidades y su repercusión en Argentina”. Revista Debate Universitario. Vol. I No 1, pp. 30-78. 2012. ISSN 2314-1530.

Tabla 8. Comportamiento de las variables dependiente e independiente.

\begin{tabular}{|c|c|c|c|}
\hline & $\mathbf{2 0 1 0}$ & $\mathbf{2 0 1 4}^{1}$ & $\mathbf{2 0 1 6}^{2}$ \\
\hline Sistema de Gestión & $\begin{array}{l}\text { La gestión no era por pro- } \\
\text { cesos ni estaba orientada a } \\
\text { obtener categorías superiores } \\
\text { de acreditación }\end{array}$ & $\begin{array}{l}\text { El sistema de gestión se orien- } \\
\text { ta a obtener categorías supe- } \\
\text { riores de acreditación }\end{array}$ & $\begin{array}{l}\text { El modelo de gestión uni- } \\
\text { versitaria por procesos para } \\
\text { asegurar la calidad de la } \\
\text { universidad se implanta }\end{array}$ \\
\hline Evaluación externa & $\mathrm{D}$ & $\mathrm{C}$ & $\mathrm{B}$ \\
\hline
\end{tabular}

1 La evaluación externa se realizó en el 2013 pero los resultados de la categoría obtenida se conocieron en el 2014.

2 La evaluación externa se realizó en el 2015 pero los resultados de la categoría obtenida se conocieron en el 2016. 
[5] F. Borrás y F. Ruso. "Capital intelectual: visión crítica y propuestas para organizaciones cubanas". Ed. UH. La Habana, Cuba: Universidad de la Habana. 2015. ISSN 0253-9276.

[6] CEAACES. "Resolución No 002-05CEAACES-2013. Reglamento para la Evaluación Externa de las IES". San Francisco de Quito, D.M, Ecuador. 2013.

[7] CEAACES. "Resolución 198-CEAACESSE-13-2015. Adaptación del Modelo de Evaluación Institucional de Universidades y Escuelas Politécnicas 2013 al proceso de recategorización de Universidades y Escuelas Politécnicas 2015”. Quito, Ecuador. 2015.

[8] J. Dias. "Calidad, pertinencia y responsabilidad social de la universidad latinoamericana y caribeña”, pp. 2-26. Enero 2017. URL: www. oei.es/ historico/salactsi/CAPITULO_03_ Dias_Sobrinho.pdf

[9] D. Docampo. "Rankings internacionales y calidad de los sistemas universitarios". Revista de Educación (número extraordinario), pp. 149-176. 2008. ISSN 1988-592.

[10] N. Fernández. "La educación y la universidad que necesitamos en América Latina: hacia nuevas políticas y nuevos modelos de gestión". XI Congreso Internacional del CLAD sobre la reforma del Estado y de la Administración Pública. Ciudad de Guatemala, Guatemala. 2006.

[11] R. Fernández, Y. Martínez y N. Velasco. "Indicadores y Estándares Internacionales de Calidad Universitaria”. Revista Calidad en la Educación. Vol. 25, pp. 17-29. 2006. ISSN: 0717-4004.

[12] E. García. "Los Rankings Mundiales de Universidades. D "objeto obscuro del deseo" a enfoques más equilibrados. Visión Ucevista". Diciembre 2016. http://www. carmengarciaguadilla.com/articulos/2007_ Rankings_Mundiales.pdf

[13] L.E. González y O. Espinoza. "Calidad en la Educación Superior: Conceptos y Modelos". Revista Calidad en la Educación. Vol. 28, pp. 244-276. 2008. ISSN: 0717-4004.

[14] C. Hernández. "Diseño del sistema de gestión de publicaciones científicas en una universidad cubana y su aplicación en la Facultad de Ingeniería Industrial de la Cujae". Tesis para optar título de Master en Ciencias de la Dirección. Instituto Superior Politécnico José Antonio Echeverria. 2014.

[15] K. Krüger y A. Mola. "Ranking de universidades españolas". Revista electrónica de recursos en internet sobre geografía y ciencias sociales. Vol. 136. 2010. ISSN 1578-0007.

[16] T.V. Moreira. "Diseño de un sistema de gestión de la Facultad de Ingeniería Agrícola del caso de estudio: proceso de vinculación con la comunidad". Tesis para optar título de Master en Ciencias de la Calidad. Universidad Tecnológica de La Habana "José Antonio Echeverria”. La Habana, Cuba. 2016. 